ÉGYPTE monde arabe

\section{Égypte/Monde arabe}

3 | 2006

Terrains d'Égypte, anthropologies contemporaines

\title{
L'art de le dire. Une réflexion méthodologique sur les histoires de djinns et autres sujets
}

\section{Barbara Drieskens}

Traducteur : Vincent Battesti

\section{OpenEdition}

\section{Journals}

Édition électronique

URL : https://journals.openedition.org/ema/1078

DOI : 10.4000/ema.1078

ISSN : 2090-7273

Éditeur

CEDEJ - Centre d'études et de documentation économiques juridiques et sociales

Édition imprimée

Date de publication : 31 décembre 2006

Pagination : 61-78

ISSN : 1110-5097

Référence électronique

Barbara Drieskens, «L'art de le dire. Une réflexion méthodologique sur les histoires de djinns et autres sujets », Égypte/Monde arabe [En ligne], 3 | 2006, mis en ligne le 08 juillet 2008, consulté le 07 juillet 2022. URL : http://journals.openedition.org/ema/1078; DOI : https://doi.org/10.4000/ema.1078 


\section{Barbara DRIESKENS}

\section{L'ART DE LE DIRE}

\section{UNE RÉFLEXION MÉTHODOLOGIQUE SUR LES HISTOIRES DE DJINNS ET AUTRES SUJETS ${ }^{1}$}

L es Cairotes adorent conter des histoires et les écouter, mais ces histoires ne sont pas des contes de fées. Les histoires dont il est question ici ont très peu à voir avec les Mille et une nuits et ne ressemblent pas aux fictions allégoriques que Watson (1992) entendit parmi les femmes de la Cité des morts². Presque tous les événements de la vie quotidienne peuvent se changer en histoire : narrer l'intrigue d'un film, rapporter une chose lue dans le journal, une chose entendue, vue, vécue. Certaines des histoires de cette contribution parlent aussi d'esprits et de forces invisibles, mais ceux qui les racontent prétendent le plus souvent qu'elles sont vraies.

Certains auteurs ont utilisé les récits recueillis sur le terrain comme une source riche d'informations. Toutefois, les histoires cairotes posent un problème sur le plan de l'information et de la véracité. Evelyn Early ou Uni Wikan ont attiré l'attention sur les différences entre I'histoire racontée et l'événement même. Early (1993, p. 24) explique comment elle élimine les exagérations et atténue l'image très positive que le narrateur donne de lui-même en confrontant ces histoires à ses observations. Wikan (1996, p. 9), quant à elle, recherche les similitudes entre les différentes versions entendues et utilise I'observation comme un outil de vérification du contenu de ces récits.

Je fus confrontée sur mon terrain à des difficultés particulières concernant les récits. Je n'écrivais pas une ethnographie générale d'un quartier ou d'un groupe

1. Traduit de I'anglais par Vincent Battesti.

2. Les histoires de Guha le fou, très populaires auprès des adultes comme des enfants, sont une exception. Ses histoires me furent souvent racontées durant mon terrain. Voir aussi Hejaiej (1996) pour des narrations de style plus féerique en contexte tunisien. 
restreint de personnes, mais je voulais enquêter sur la place réservée aux djinns dans la vie quotidienne des Cairotes de la classe moyenne inférieure ${ }^{3}$ (Drieskens, inédit). Les djinns sont invisibles et créés de feu, ils vivent parmi les humains, en particulier dans les endroits humides comme les salles de bains et sombres comme les escaliers. Ils ne sont pas essentiellement mauvais, mais ils sont imprévisibles. Lorsqu'ils se mettent en colère ou tombent amoureux d'un être humain, ils peuvent le posséder et provoquer des douleurs, des insomnies, des cauchemars et d'étranges comportements. Ils se manifestent à travers certains symptômes dont souffrent leurs victimes ou bien en rêves ou bien encore lors de rituels de divination. II n'est pas difficile de comprendre qu'une vérification empirique de ces récits sur les djinns est impossible pour un anthropologue qui n'est ni devin ni possédé. De plus, deux versions d'un même récit de possession peuvent tellement différer qu'il devient difficile d'y reconnaître les mêmes événements et personnes.

II me fallait donc développer une approche de ces récits qui ne soit ni réductrice ni déductive. Je ne souhaitais pas les réduire à la seule expression de tensions sociales, comme expression symbolique et symptomatique de l'indicible ${ }^{4}$. D'autre part, si j'éliminais les contradictions, les dissonances, les exagérations et les invraisemblances, il ne restait plus grand-chose de ces récits. II n'était pas possible de dissocier les inventions et les rajouts personnels de la réalité vécue du narrateur ni de séparer l'aspect surnaturel de la possession de la souffrance réelle du possédé. J'ai donc développé une approche globale, qui intègre non seulement le contenu des histoires, la description des djinns, mais aussi la totalité de l'événement narratif. Pour expliquer la richesse de ces histoires, il était nécessaire d'en considérer à la fois le contenu et la forme, mais surtout de penser la rencontre entre narrateur et auditeur. Dans une perspective deleuzienne, j'ai abordé ces histoires par l'entre-deux de la rencontre ${ }^{5}$. Ma première question n'était pas «quel est le réel qui se cache derrière ces histoires? ", mais « que se passe-t-il quand quelqu'un raconte une histoire à propos de djinns?».

Raconter une histoire est un art qui implique à la fois les auditeurs et le narrateur. Il serait faux de distinguer entre un narrateur actif et un récepteur passif. Le bon auditeur n'est jamais passif et écouter n'est pas une simple activité de réception. Le public et le narrateur participent tous deux à l'événement de narration. Ensemble,

3. Ces hommes et femmes travaillent, le cas échéant, principalement comme fonctionnaires ou employés d'entreprises récemment privatisées. Leurs salaires sont bas, augmentés de bakchichs et du soutien de membres plus aisés de la famille. C'est un groupe très diversifié, certains possèdent des diplômes, d'autres quasiment aucun, mais la plupart savent lire et écrire. Sur la question des classes sociales en Égypte, voir Inhorn (1996, p. 34-40).

4. Devisch, Stuert et Verrept (1989) sur le lien entre la symbolique et la physiologie.

5. Voir Deleuze et Parnet (1991, p. 90-91). La rencontre n'est pas abordée dans la perspective de la réunion de deux éléments, $A$ et $B$, mais dans celle de l'entre-deux, qui n'équivaut pas à la somme de $A$ et de $B$, mais qui est en devenir. 
ils constituent le sujet, le contexte opportun, la forme de l'histoire et les émotions en jeu ${ }^{6}$. Les histoires naissent d'une rencontre, elles sont adaptées à un contexte, à des participants, à un lieu et à un moment. Dans cette contribution, je montrerai comment tous ces éléments sont en corrélation et pourquoi tous ces éléments doivent être pris en compte pour saisir la richesse, les multiples messages et le sens d'une histoire. C'est pourquoi j'ai aussi inclu l'anthropologue dans I'analyse, car, de la même manière que le narrateur et le public ne sont pas simplement actifs et passifs, il n'y a pas non plus de sujet spontané et authentique et d'observateur silencieux et objectif, surtout lorsqu'il s'agit d'histoires ${ }^{7}$.

\section{QUESTIONS ET HISTOIRES}

\section{Poser la question des Djinns}

Quand j'ai débuté mon terrain en $1998^{8}$ sur les pratiques et les croyances concernant les créatures invisibles parmi la classe moyenne inférieure cairote, je dus faire face à l'évident contraste entre la richesse des histoires racontées entre voisins et amis et la rareté et l'austérité de mes propres conversations et discussions qui reposaient essentiellement sur le jeu de questions/réponses. L'un des problèmes principaux était que le thème des djinns, au Caire, est très ambigu. Cette ambiguïté tient d'abord à la nature équivoque des djinns. Selon le Coran, quand Allah créa le monde, il créa trois types d'êtres intelligents : les humains formés d'argile, les anges de lumière et les djinns d'un feu sans fumée. Les djinns ne sont pas mauvais, mais a-moraux : ils ne font pas la distinction entre le bien et le mal, ils aiment les plaisirs de ce monde et tentent d'inciter les humains à s'abandonner à leurs désirs. Les djinns sont souvent associés aux démons (shayâtîn) même s'ils en diffèrent.

L'existence des djinns ne peut être niée, car le Coran la confirme. Pour beaucoup de musulmans cairotes ${ }^{9}$, toutefois, la possession et toute autre façon

6. Voir Frishkopf (1996) sur l'importance et l'effet structurant du feed-back en poésie soufie.

7. Voir Céfaï (2003, p. 555) pour qui «Seule une méthodologie positiviste peut croire que l'enquête consiste en un simple transfert d'informations objectives d'un enquêté vers un enquêteur ».

8. Mon terrain dura de septembre 1998 à décembre 2001, mais je suis restée au Caire durant la phase de rédaction de ma thèse jusqu'en avril 2003, ce qui me permettait une vérification constante des données.

9. J'ai effectué mon terrain parmi les musulmans, mais des pratiques très similaires existent parmi les coptes du Caire. D'ailleurs, les guérisseurs coptes bénéficient d’une bonne réputation d'exorcistes autant parmi les patients coptes que musulmans. Voir Drieskens (2004) au sujet de l'arrestation d'un guérisseur copte. 
d'être en commerce avec des djinns supposent l'impureté et une religiosité insuffisante. Être possédé ou n'être même qu'influencé par les djinns est une marque de faiblesse. Les victimes faciles sont des personnes insuffisamment pieuses et pures. Cette image négative de la plupart des pratiques liées aux djinns est renforcée par la diffusion d'enseignements islamiques « réformés ${ }^{10}$. Ces réinterprétations du Coran et d'autres sources islamiques reconnaissent l'existence des djinns, mais les pratiques liées à ces créatures invisibles sont sujettes à de vigoureux débats moraux. Ce discours islamique récent introduit de nouvelles distinctions entre les voies correctes et les voies illicites pour parler des djinns ${ }^{11}$ et pour interagir avec eux. La divination et les rituels de guérison visant à réconcilier les djinns et la personne affligée (zâr $r^{12}$ ) sont condamnés comme des formes de superstition, voire d'infidélité et d'idolâtrie.

Une autre raison qui explique que les gens évitent de parler ouvertement de leurs expériences avec les djinns est que cette croyance, la possession, et surtout les pratiques qui lui sont associées sont considérées comme un signe d'ignorance et d'arriération. Les rituels prophylactiques du quotidien sont jugés comme des habitudes vieillottes et niaises et les rituels de possession et de divination au moyen de djinns sont dépeints comme chimériques et tenant du charlatanisme. Parler des djinns n'est donc jamais neutre : cela implique toujours pour le narrateur un positionnement moral et son jugement par le public.

Les raconteurs d'histoires adoptent habituellement un langage métaphorique pour évoquer les djinns, non seulement pour occulter le sujet de leurs discussions, mais aussi à cause du risque de parler de "ceux-là ». Il suffit de les mentionner pour les rendre présents, pour les inviter dans la pièce et

10. Les classes moyennes inférieures semblent les plus sensibles à la nouvelle tendance en islam, al-sahwa al-islâmiyya, qui est largement publicisée par des prêcheurs à la télévision, par les magazines et par d'autres médias. Cette doctrine récupère les croyances populaires et leur donnent une forme restreinte, perçue comme plus vraie, plus islamique et plus moderne. Voir Hanafi (1982), Starret (1998), Mahmoud (2001) et Hirschkind (2001).

11. Voir I'observation d'Abu-Lughod (1997, p. 529) chez les Bédouins du désert libyque: "When one considers the uses of religious talk in Awlad Ali conversations and narratives, two things become clear: the religious discourse is always in tension with others, and it accomplishes complex ends in the narratives and conversations in which it is inserted ».

12. Au sujet de ces rituels complexes de guérison visant à la réconciliation entre djinn et être humain - et non pas à l'exorcisme -, voir Abdelsalam (1994 et 1995), Boddy (1989), Böhringer-Thärigen (1996), Constantinides (1977), Kenyon (1995), Morsy (1978), Nabhan (1994), Saunders (1977), Sengers (2000). Le zâr est habituellement dépeint comme un moyen inacceptable de traiter la possession, contrairement à I'exorcisme (guérison avec l'aide du Coran). Voir Schöller (2001) pour une description de l'exorcisme. 
les inciter à s'immiscer dans les affaires humaines. Les narrateurs usent donc de périphrases et, pour les comprendre, il est nécessaire d'être familier de la question et de sa terminologie.

La plupart du temps, soit mes interlocuteurs se référaient au Coran pour de «vraies» informations sur les djinns, afin de paraître comme de bons musulmans, soit ils déniaient simplement la réalité de ces histoires et s'affirmaient alors comme des citadins rationnels et modernes. S'ils répondaient à mes questions, ils essayaient de rester aussi proches que possible du discours public islamique moderne, mais la plupart du temps ils me renvoyaient vers les «spécialistes ». Si ce spécialiste était un marginal (femme, vieux, populaire (baladî) et/ou possédé), ce qu'il/elle racontait était présenté comme de simples plaisanteries. La plupart du temps, on me renvoyait vers un cheikh, c'est-à-dire un homme, religieux, respecté et instruit, qui avait autorité pour parler de ces choses et pouvait m'enseigner la « vraie vérité ».

\section{Histoires accidentelles}

Ce n'était pas seulement parce qu'ils craignaient pour leur réputation ou parce que je ne posais pas les bonnes questions que l'on ne me racontait pas d'histoires. Tant que je m'efforçais d'interférer aussi peu que possible, tant que je $m^{\prime}$ efforçais de minimiser ma présence d'observatrice étrangère, je ne représentais pas un bon public. Mes préoccupations professionnelles et éthiques sur la question de l'objectivité faisaient de moi un auditeur vraiment ennuyeux. Tant que je n'avais pas compris cela, je devais attendre qu'une personne raconte à une autre une histoire de djinns et qu'accidentellement je sois là.

Les soirées d'été dans les ruelles formaient le cadre idéal des histoires, qui portaient alors principalement sur la famille et les voisins, la maladie et la mort, le mariage, etc. Très peu de ces histoires traitaient des djinns, car un narrateur préfère toujours éviter un jugement inopportun sur sa moralité, sa religiosité ou sa rationalité. L'ambiguïté de la question des djinns rend difficile la narration d'histoires de djinns ou de possession liées à des expériences personnelles d'une façon telle que le récit soit crédible, acceptable et en même temps adapté à un large public. Cela exige que l'assistance soit relativement homogène et, de fait, elles sont narrées à l'intérieur de la maison pour un public restreint.

La plupart des auditeurs considèrent les anecdotes et les histoires sur les djinns comme inutiles, comme de vaines paroles (kalâm fârigh), sauf s'il s'agit de l'expérience personnelle du narrateur. Dans ce cas, le narrateur peut prétendre avoir autorité sur le sujet, mais cette implication personnelle est toutefois maintenue secrète au sein de la famille afin d'éviter les jugements inopportuns à l'extérieur. C'était en cuisinant, en gardant les enfants et en buvant sans fin du thé que j'entendis mes premières « vraies » histoires de djinns. Au début, je pensais qu'elles m’apprendraient de manière simple et directe la réalité des djinns, qu'elles m'informeraient sur leur rôle dans la vie quotidienne de mes informateurs. 


\section{DE QUOI PARLENT LES HISTOIRES?}

Une après-midi, alors que j'étais assise avec Huda dans son appartement à 'Izbatal-Sa $̂ \hat{i d a}^{13}{ }^{13}$ Nafîsa, une voisine, entra pour téléphoner. Elle était curieuse de savoir qui j'étais et pourquoi je venais ici. Quand je lui ai expliqué le sujet de mes recherches, elle a semblé enthousiasmée : «J'en ai un en moi! » me sourit-elle. "Presque toutes les nuits, ma cheikha [un djinn féminin et religieux, presque un ange] me rend visite. Elle est habillée de blanc, porte un voile et son nom est 'Â'isha ». Elle nous raconta que 'Â'isha lui avait apporté de l'or, de l'argent, des fruits pendant qu'elle dormait, mais que du jour où elle confia à sa mère l'origine de ces cadeaux, le djinn cessa de lui être agréable et commença à l'embêter et à la torturer. Huda avait refusé de me parler de djinns depuis plus d'un mois et chaque fois qu'une autre personne voulait me parler, elle les décourageait d'une remarque sceptique. Elle demanda à Nafîsa : "Si cela te fait mal, pourquoi n'exorcises-tu pas ce djinn? ». Nafîsa parut surprise et un peu un peu effrayée, elle grommela qu'il fallait en discuter avec Sayyid, le frère de Huda, puis disparut.

Une histoire est souvent autre chose que ce qu'elle semble être à première vue. Lorsque Nafîsa quitta la pièce, les commentaires de Huda me rendirent perplexe. "Tout cela sont des kalâm fârigh (vaines paroles), une blague. Tu ne devrais pas croire un mot de ce qu'elle dit. C'était très drôle de te voir prendre ça au sérieux! As-tu vraiment cru qu'elle te disait ça pour être utile à ta recherche? »Chaque fois que je mentionnais I'histoire de Nafîsa, Huda me donnait une nouvelle interprétation : «Elle voulait juste te montrer comment elle était bien avec sa cheikha. Elle voulait attirer ton attention pour te séduire et devenir ton amie. Elle voulait vérifier si tu avais vraiment appris quelque chose sur les djinns dans tes études ». En d'autres occasions encore, Huda semblait croire que l'histoire lui était destinée : «Nafîsa voulait me faire croire qu'elle était possédée et ainsi s'excuser de son comportement parfois étrange. Elle espérait que je parlerais à mon frère de sa belle djinniyya religieuse ».

\section{Intentions du narrateur et interprétations des auditeurs}

Ce n'est qu'après la visite de Nafîsa que j'appris que Sayyid connaissait bien les djinns et qu'il avait des capacités de guérison. Huda ne voulait pas que je le sache pour ne pas que mon jugement sur elle et sa famille soit influencé et pour éviter qu'à travers mon étude ils apparaissent sous un mauvais jour. Ses préoccupations se transformèrent pour moi en une lourde responsabilité, certaines choses ne devaient pas être publiées, je devins la gardienne de sa réputation, de celle de Sayyid et sa famille, du voisinage, du quartier et des

13. 'Izbat-al-Sâîda est I'une des parties les plus vieilles du quartier d'Imbaba, situé à l'ouest et au nord-ouest du Caire près de la rive gauche du Nil. Trois millions de personnes vivent pour la plupart dans des immeubles informels. 
Égyptiens en général. L'écriture de ma recherche sur «Vivre avec les djinns » (Living with Djinns) demandait de conserver un équilibre délicat entre l'intérêt scientifique et le respect de mes informateurs ${ }^{14}$.

Une fois mise au courant des capacités de guérisseur de Sayyid, ma présence fut acceptée quand, deux semaines plus tard, Nafîsa demanda conseil à Sayyid pour se débarrasser de son djinn. Elle lui parla des sept djinns qui la possédaient, de ses souffrances, des différents symptômes et des formes terrifiantes que prenaient les djinns quand ils apparaissaient dans ses rêves. II me semblait contradictoire d'être possédé par un djinn femelle en une occasion, puis par sept mâles en une autre. Quand je questionnai Nafîsa sur sa cheikha, elle m'affirma que les sept djinns étaient les assistants de 'Â'isha. Elle ajouta plus de détails tendant à prouver la véracité de ses dires. Elle désigna ses boucles d'oreille en expliquant que 'Â'isha lui avait apporté de l'or et des cadeaux. "Demande à ma mère! » disait-elle quand elle racontait comment de terreur elle se réveillait en pleine nuit après avoir vu les djinns dans ses rêves. J'avais l'impression que la concordance entre le contenu de l'histoire et ce qui s'était réellement passé était secondaire dans certains récits ${ }^{15}$.

L'histoire de Nafîsa changeait au gré des circonstances et de l'auditoire. La première fois, Nafîsa racontait l'histoire de sa possession par la cheikha. Probablement espérait-elle ainsi attirer mon attention et devenir mon amie. Une autre fois, elle me raconta l'origine de sa possession: son mari l'avait fait pleurer la nuit à la suite de quoi elle s'était évanouie dans la salle de bain. Cette fois, en insistant sur ses souffrances et les problèmes avec son mari, je sentis qu'elle espérait que je la prenne en pitié. Par contre, quand Huda était présente, Nafîsa mettait l'accent sur les formes de la possession par ce djinn qu'elle présentait comme une cheikha, soulignant sa religiosité, parce qu'un djinn (bon) musulman entraîne moins de tort pour sa réputation qu'un mauvais. Enfin, en la présence de Sayyid, le guérisseur, elle parlait de ses sept djinns pour que le sérieux de son tourment l'impressionne.

Nafîsa disait à Sayyid que son djinn et ses sept assistants étaient très forts, car elle voulait qu'il croit qu'il était impossible de l'exorciser. Pour Sayyid, elle était effrayée à l'idée de "perdre » ses djinns qui lui permettaient d'excuser son comportement étrange ${ }^{16}$ et elle craignait leur vengeance si l'exorcisme

14. Céfaï (2003, p. 612) note que « Tout n'est pas bon à écrire. L'enquêteur doit choisir les informations à divulguer, parfois par diplomatie, pour ne pas ternir le portrait d'un homme public ». Cela reste vrai à des niveaux micro où la relation entre anthropologue et informateur repose sur la confiance mutuelle.

15. Abu-Lughod (1997, p. 612) avance un argument similaire pour les Bédouins du désert libyque.

16. Parfois, Nafîsa quittait la maison sans prévenir et marchait pendant des heures, disparaissant parfois pendant des jours, après quoi sa famille la retrouvait généralement près du tombeau d'un saint. Nafîsa semblait ne jamais se souvenir de ce qui était arrivé. 
échouait. Mais les deux interprétations de Sayyid relevaient d'une logique différente. La première suggérait que les djinns n'étaient qu'une excuse; la seconde reconnaissait leur pouvoir et donc le bien fondé de la peur de Nafîsa.

L'histoire changeait en fonction des circonstances et des auditeurs; puis chacun interprétait I'histoire diversement. Nous savions que I'histoire était réorientée et réadaptée à chaque fois et nous tentions de lire au travers et au-delà de l'image que le conteur essayait de donner. Les commentaires des auditeurs suggéraient que diverses versions d'une histoire pouvaient être entendues comme différentes formes de manipulation. Sayyid, Huda et moi avions tous le sentiment d'être manipulés, mais chacun de nous en donnait une interprétation différente. Pour pleinement saisir la valeur d'une histoire, il ne faut donc pas s'arrêter à sa vérité de référence ou ne s'intéresser qu'à son contenu évident, mais il faut accorder autant d'importance aux intentions du narrateur qu'aux réponses du public.

\section{Véracité des faits ou sagesse de la morale?}

Dans la plupart des cas, les auditeurs ne considéraient pas particulièrement pertinent d'essayer de savoir ce qui s'était réellement passé dans la réalité. Même si les références au réel étaient souvent biaisées, le savoir qui se transmettait à travers le récit pouvait néanmoins encore être pertinent et juste. Cela signifie qu'il pouvait s'agir d'une sagesse morale ou un conseil judicieux transmis sous forme d'histoire. Le récit de Umm Ahmad sur la visite de son mari à son frère 'Âtî en est une bonne illustration. Il ne s'agit pas de djinns, mais cette histoire $\mathrm{m}$ 'a été racontée comme une leçon de générosité.

Mon mari rendit visite à son frère 'Âtî qui l'avait invité à dîner. La femme de 'Âtî lui servit à manger, mais elle ne lui apportait que de petites quantités dans de petites assiettes. Mon mari, Abû Ahmad, était un homme grand et bien portant et il mangeait habituellement en grandes quantités. Il revint à la maison le ventre creux, choqué par le traitement que sa belle-sœur lui avait réservé. Il se plaignit de son avarice à sa mère qui lui transmit le message. Lorsqu'il rendit à nouveau visite à 'Âtî, sa femme, pour se venger, apporta toute la marmite sur la table de manière provocante. La mère de 'Âtîn'aimait pas la femme de 'Âtî, ni aucune de ses belles-filles, moi excepté (Umm Ahmad), parce qu'elle savait que j'étais généreuse, que ma maison était toujours ouverte et que mon hospitalité était réputée. Tout le monde aimait me rendre visite pour partager mes plats toujours copieux et goûteux.

Dans cette histoire, Umm Ahmad se dépeint comme une femme généreuse, mais elle démontre aussi la valeur de la générosité et de l'hospitalité en général. Son intention principale était de m'enseigner ces valeurs et de m'indiquer la bonne conduite à tenir. Beaucoup des femmes que j'ai rencontrées au Caire 
préféraient à l'exhortation, l'enseignement et le conseil par le biais d'histoires. Une histoire permet de formuler des remarques de façon indirecte, ce qui rend la critique plus facile à accepter pour l'auditeur. Ensuite, les histoires sont plus faciles à mémoriser : les femmes récitent souvent des histoires qu'elles tiennent de leur mère. Enfin, l'effet d'une histoire est plus fort, car l'auditeur est émotionnellement impliqué dans ce qui est dit; à cet égard la forme de la narration est essentielle.

\section{Transmettre des émotions}

Pour marquer la rupture entre une histoire et d'autres formes discursives, le narrateur utilise une phrase introductive particulière, un changement de ton, d'attitude, certains mouvements des mains... Ce qui différencie une histoire $d^{\prime}$ autres formes de discours est le haut niveau d'interaction et d'attention des participants, la densité des messages et des informations véhiculées et l'escalade des émotions par les exclamations et les commentaires, même sur de très courtes histoires. Raconter des histoires a ceci de particulier que le narrateur prend le contrôle de la rencontre, qu'il dirige les réactions des autres.

Il construit son histoire sur des répétitions, qui soulignent son propos, mettent I'accent sur certains éléments et soutiennent I'attention de l'interlocuteur. Les encouragements des auditeurs sont aussi importants que les mots du narrateur. Un bon narrateur doit interrompre ses phrases, en laissant un silence, pour offrir à ses auditeurs une chance de participer, de réagir, de lui demander de poursuivre. Il est important de "ne pas vendre une histoire au rabais », ce qui signifie qu'il ne faut pas raconter une histoire quand les conditions ne sont pas réunies pour qu'elle soit appréciée à sa juste valeur ou pour qu'elle déclenche l'effet désiré. Les commentaires des auditeurs encouragent l'orateur à poursuivre, mais créent également un contexte qui modifie I'histoire elle-même. Ainsi, nous pouvons dire que les histoires sont construites de façon intersubjective.

Pendant son récit, le narrateur recourt fréquemment à des gestes et des mouvements permettant de visualiser les faits. Les expressions de son visage évoquent les émotions des personnages, les mouvements de ses mains et de ses bras rendent compte de leurs actions. Cela permet à l'auditoire de s'identifier plus facilement avec les différents personnages de l'histoire. Dans son récit, Umm Ahmad montrait avec ses mains la grande taille de son mari, la petitesse des assiettes que la femme de 'Âtî lui apportait, son impolitesse dans sa façon de déposer la marmite sur la table. Elle souriait pour montrer sa propre générosité et sa propre satisfaction d'être appréciée par sa belle-mère. De la même manière que les gestes, les intonations de la voix permettent de communiquer avec le public et de transmettre des émotions : la colère, I'indignation, la joie... Dans certains passages de son récit, Umm Ahmad murmurait presque et les personnes présentes devaient alors se concentrer pour l'entendre, puis elle élevait la voix d'un coup, comme pour les réveiller. En d'autres passages, elle ralentissait le débit des mots comme pour souligner chacun d'eux, puis elle accélérait pour que son public ne perde pas le fil de l'histoire. 
Dans la narration, les émotions se succèdent, riches et diverses, l'étonnement est suivi de l'horreur, la tristesse succède à la pitié et alterne avec la joie. Les émotions passent des personnages de l'histoire aux auditeurs, par le mécanisme de l'identification, dans un dessein précis. Umm Ahmad n'aimait pas mon habitude de ne préparer que juste ce qu'il fallait de nourriture pour les personnes présentes. Dans la première partie de l'histoire, elle m'accuse indirectement d'avarice par identification avec la femme de 'Âtî (nous servions toutes deux de petites portions). Dans la suite de I'histoire, Umm Ahmad déplace habilement I'identification vers un autre personnage, elle-même (si je lui ressemblais tout le monde me rendrait visite). La première partie engendre un sentiment de honte, suivie par la désapprobation et l'indignation. Dans la seconde partie, elle crée une atmosphère de complicité par son sourire et transmet la joie d'être aimée. Dans l'histoire, les personnages subissent l'avarice de la femme de 'Âtî et sont ravis par la générosité d'Umm Ahmad. La morale est évidente : I'avarice est mauvaise, la générosité est bonne. L'auditeur s'identifie d'abord avec la femme de 'Âtî, puis avec Umm Ahmad. Avec ce déplacement d'identification, le narrateur adoucit sa critique et tente d'enseigner la générosité.

Avec les histoires, les Cairotes utilisent le pouvoir de la parole sur le cœur pour le transformer. Ceci est comparable à ce qu'Hirschkind (2001, p. 623-649) écrit au sujet des histoires et des discours religieux. Les mots sont utilisés pour toucher le cœur des croyants, ils sont choisis à cause de leur pouvoir à inciter à la vertu, non pas en faisant appel à la raison, mais en utilisant une compréhension émotionnelle. Les discours religieux, qui visent la manipulation, jouent principalement sur la peur, l'humilité, le remords, la repentance et la sérénité.

"Un bon auditeur écoute avec son cœur »: dans le raisonnement des Cairotes, la distinction entre la raison et le cœur ne correspond pas exactement à l'opposition entre sentiment et réflexion, émotion et raison. Le cœur transcende la raison, qu'il inclut, et la compréhension émotionnelle est beaucoup plus profonde, car elle a un impact immédiat qui dépasse l'analyse réflexive. Les histoires ont le pouvoir de changer ceux qui les écoutent. Si une histoire ne provoque pas de changement, si elle n'affecte pas son public, c'est un échec. Ce n'est pas seulement de la manipulation de la part du narrateur, comme Nafîsa qui voulait inspirer la pitié et qui demande de l'aide, mais c'était aussi qu'une bonne histoire doit inculquer de bonnes vertus comme la générosité.

La narration d'une histoire affecte non seulement I'auditoire, mais transforme aussi le conteur par la succession des émotions évoquées. Wikan parle de l'importance pour les Cairotes de la classe moyenne inférieure d'exprimer les choses avec leur corps ("talk things out of their body », Wikan, 1996, p. 22-23). Early (1982 et 1985, p. 179; 1993, p. 11) fait aussi mention des effets thérapeutiques des histoires, qui offrent un certain soulagement en permettant de partager des expériences traumatiques ou difficiles, mais pas nécessairement autobiographiques puisque la même catharsis peut être obtenue avec $n^{\prime}$ importe quelle bonne histoire. 


\section{Manipulation}

Le récit d'une histoire prend toujours la forme d'une rencontre entre le conteur, avec ses intentions, et les auditeurs, qui ont un rôle tout aussi actif. Le conteur se préoccupe souvent d'abord de sa réputation, de la justification de ses actes. Il présente une certaine image de lui-même et attend des autres qu'ils le considèrent en conséquence (Wikan, 1996, p. 7). Certains utilisent les histoires à des fins pratiques, pour que l'auditoire ait pitié et qu'il prête de l'argent ou propose son aide. D'autres visent une manipulation morale, comme dans I'histoire de la femme de 'Âtî.

Une autre question importante qui se pose à propos de la narration est de savoir si le narrateur offre réellement des informations ou si, plutôt, il n'en recherche pas. Souvent, mes informateurs me racontaient des histoires pour découvrir ce que je faisais, ce que je savais des djinns. Ils voulaient savoir si je pouvais être utile en recommandant un guérisseur, en reconnaissant leur possession et en confirmant leur histoire. Parce que la narration est interactionnelle, il est difficile pour l'auditeur de cacher son opinion, de conserver sa distance et de dissimuler ce qu'il sait. C'est pourquoi les Cairotes disent qu'un bon conteur peut gagner plus que ce qu'il donne au cours de la narration ${ }^{17}$.

Par ailleurs, I'attitude de l'auditoire est un phénomène complexe. Parfois il accepte simplement le message, d'autres fois il le comprend différemment de ce que recherchait le narrateur. Certains auditeurs trouvent dans une histoire un message et une morale que le narrateur n'avait pas l'intention de faire passer.

Une femme m'avait raconté être possédée et combien elle en souffrait. Le djinn la torturait d'une cruelle manière et, à plusieurs occasions, elle était allée voir un guérisseur pour exorciser l'esprit, mais le traitement ne réussissait jamais. Chaque fois le djinn revenait, plus brutal et plus en colère qu'avant. Son histoire pouvait être considérée comme un moyen d'expliquer son comportement, comme une justification pour ses colères hystériques et incontrôlées. Pour l'un des auditeurs, toutefois, comme il l'expliqua plus tard, la vraie morale de cette histoire était de montrer le mal de l'avarice. II avait observé combien cette dame semblait pauvre en comparaison de sa voisine. Alors que son mari avait un bon travail avec un bon revenu, alors que lui et ses enfants étaient bien et proprement habillés, elle, au contraire, ne portait ni bijoux, ni maquillage, seulement des vêtements bon marché. Donc, selon cet auditeur, le vrai sujet de l'histoire était la haine refoulée de cette femme pour son pingre de mari. La morale que l'on pouvait en tirer était que l'avarice semait la haine entre les gens.

On dit qu'écouter des histoires est un art autant que de les raconter. L'art de distinguer, de comprendre autant de messages que possible, de partager les émotions avec le narrateur. Une fois, Umm Sayyid, la mère de Huda, m’a

17. Cette pratique peut être comparée aux jeux de mot décrit par Gilsenan pour le Liban (1993, p. 158). 
vu prendre des notes après qu'une voisine m'avait raconté une histoire. Elle m'a demandée: "N'as-tu donc rien compris? As-tu réellement pensé qu'il s'agissait de djinns?».

\section{À travers les apparences}

Les apparences (manzar) ont une importance essentielle dans la société cairote. Ce que les gens disent, ce qu'ils peuvent penser, ce qu'ils voient et comment ils jugent le comportement des autres, déterminent dans une grande mesure les choix d'une personne. Hommes ou femmes se présentent de manière à paraître tels qu'ils veulent que les autres croient qu'ils sont, s'attendant à être traités comme tels ${ }^{18}$. Ils ajustent leur présentation de soi au jugement présupposé des autres. Dans le même temps, ils essayent toujours de lire à travers les apparences des autres et de juger dans quelle mesure elles coïncident avec la réalité en arrière-plan.

Il n'est pas très difficile de voir, au moins partiellement, à travers la présentation de soi. Il suffit de maîtriser quelques connaissances pratiques, le prix de certains objets par exemple, pour avoir une idée précise du niveau de vie des autres. De plus, les apparences résistent rarement à l'incohérence de certains détails, notamment vestimentaires, et il y a des traits difficiles à cacher, comme I'accent et l'attitude. Certains ont l'intuition des caractères humains, ce qui leur permet de deviner les intentions derrière les mots et les actes.

Firâsa est l'art de lire dans le cœur, la pensée d'autrui ${ }^{19}$. C'est la capacité de déduire des informations de données verbales et non verbales. Dans le cadre de la narration, firâsa peut signifier une sensibilité spécifique aux détails, révélateurs de la condition sociale et familiale d'un narrateur et, par-là, de ses intentions ou du message derrière les mots. Cela demande d'observer le langage du corps, mais aussi la forme du visage, des mains et des pieds pour connaître son état de santé ou sa psychologie. Le concept de firâsa est aussi lié à celui de fitra, c'est-à-dire la compréhension immédiate des différentes réalités entremêlées ${ }^{20}$. Ces deux concepts sont $\mathrm{d}^{\prime}$ origine religieuse, mais mes interlocuteurs les appliquaient aux réalités sociales, particulièrement lorsqu'ils me parlaient de l'art de l'écoute. Un bon auditeur encourage le narrateur à une meilleure performance, un bon auditeur est émotionnellement impliqué dans une histoire, il saisit le sens par une intelligence émotionnelle (fitra). Bien écouter suppose également d'être capable de saisir le contexte plus large de ce qui se dit et de comprendre les intentions du narrateur, pour lire au-delà des apparences (firâsa).

18. Voir l'analyse de la présentation de soi chez Goffman (1973, p. 25).

19. Firâsa renvoie à une technique $d^{\prime}$ induction divinatoire, mais aussi à l'art ordinaire de voir au-delà des apparences. Voir Fahd (1965).

20. Voir Gobillot (2000, p. 47). 


\section{LES ÉLÉMENTS CONSTITUTIFS}

\section{Constances?}

Des auteurs comme Early (1993) et Wikan (1996) mentionnent trois phases dans leurs études des histoires. La première est la phase de l'écoute et de l'enregistrement. L'un des principaux problèmes du terrain au Caire, en particulier pour des récits spontanés, est la quasi-impossibilité d'utiliser un magnétophone. La présence d'un magnétophone fausse le contexte de telle façon que toute la spontanéité et la magie de la narration sont perdues (Early, 1993, p. 12-13).

Selon Early (1993) et Singerman (1995), les histoires sont souvent répétées. Cette répétition est la deuxième phase de leur travail, car elle offre à l'anthropologue l'opportunité de corriger ou d'ajuster ses notes. J'ai déjà souligné que les histoires changent avec leur contexte. La plupart des chercheurs se concentrent sur les constances et les similitudes entre différentes versions, avec l'idée que les éléments invariants coïncident avec ce qui s'est réellement passé. Je vais montrer plus loin que cela n'est pas nécessairement vérifié.

La troisième phase est l'interprétation du contenu de l'histoire à la lumière du contexte de sa narration et de l'événement relaté. Cela permet de nuancer les exagérations et d'atténuer les présentations de soi trop flatteuses. Cette approche en trois phases montre clairement que la visée principale de ces auteurs est le contenu des histoires. En considérant les récits comme des moyens de transmettre des informations, ils négligent d'autres niveaux de compréhension et ne considèrent pas la narration comme un art situé dans I'entre-deux du narrateur et du public.

Je présente une approche complémentaire, en deux parties, qui dérive principalement de mon travail sur les djinns. Tout d'abord, je propose de se concentrer sur les éléments constitutifs d'un récit. Ce sont les constantes dans les histoires de djinns, les idées élémentaires concernant le monde de l'invisible (al-ghayb) qui rendent crédible une histoire, car elles sont partagées entre le narrateur, son auditoire et souvent un public plus large. Deuxièmement, je m'intéresse à la pratique du récit comme rencontre, là où l'histoire prend forme, dans l'entre-deux du public et du narrateur.

Les opinions des Cairotes au sujet des djinns sont multiples, incohérentes et souvent très différentes. Nous ne pouvons pas pour autant nier qu'il existe des conceptions partagées sur les relations entre les djinns et les humains. Il y a de multiples manières de parler des djinns, mais l'on ne peut pas dire n'importe quoi et, à l'évidence, certains points de vue, certaines interprétations ne sont pas crédibles. A contrario, l'histoire de Nafîsa, qui racontait qu'elle a été possédée parce que son mari l'avait fait pleurer la nuit à la suite de quoi elle s'était évanouie dans la salle de bain, en est un exemple. II est très courant d'associer le début de la possession à un moment de crise : s'endormir en pleurant est perçu comme très dangereux et il est souvent dit que la salle 
de bain est la demeure préférée des djinns. Si les gens partagent beaucoup d'idées identiques sur le monde des djinns, c'est qu'ils ont vécu des expériences et entendu des histoires similaires.

Dans cette perspective, la religion joue bien sûr un rôle important : quand les musulmans égyptiens parlent des djinns, ils font souvent référence aux versets coraniques où les djinns sont mentionnés. Ces versets sont expliqués dans le tafsîr et les complètent des éléments des hadîth, la tradition du Prophète. Néanmoins, même si les Cairotes aiment prétendre que les sources religieuses sont les premières et principales sources pour la connaissance des djinns, l'influence de la religion n'est pas toujours si importante. En effet, les différences entre cairotes musulmans et chrétiens dans la manière de concevoir les djinns et de vivre au quotidien avec eux n'est pas si grande.

Les médias aussi influencent les manières de concevoir les djinns, mais la variété de leurs présentations et de leurs interprétations est immense. Certains journaux expriment le plus grand scepticisme, ils dépeignent les guérisseurs de campagne comme des charlatans et des escrocs qui couchent avec leur patiente pour les guérir de la stérilité (al-Naba' du 2 janvier 2000 et Akhbâr al-Yawm du 18 mars 2000). D'autres offrent des interprétations d'inspiration psychologique reliant ce phénomène à des données sur I'hypnose ou la télépathie. Mustafâ Mahmûd, le présentateur vedette d'un programme scientifique de la télévision égyptienne, comparait ainsi le zâr (un rituel de guérison traditionnel) à des électrochocs. Il le qualifiait de psychodrame visant au soulagement des tensions internes et de l'agressivité (2 mars 2002).

Outre ces sources formelles, les conceptions du monde invisible sont essentiellement façonnées par les rituels et par les pratiques du quotidien. Beaucoup de gens brûlent de l'encens le vendredi pour purifier la maison et la protéger des djinns et des mauvaises influences. Au rituel du septième jour après la naissance, la subû'a, on jette une mixture de sept graines et de sel pour apaiser «ceux de dessous terre ». Quand on passe par un passage sombre ou que I'on monte un escalier, on chuchote « dastûr yâ rabb! » pour se protéger des djinns. On dit « al-salâm 'alaykum » quand on passe le seuil d'une maison ou d'un appartement, même s'il n'y a personne à l'intérieur, pour saluer les anges et les djinns. Pour l'enquêteur, ces pratiques sont source de savoirs sur les djinns et peuvent compléter les observations empiriques. Mais c'est une source de savoir confuse et ambiguë, parce que ces pratiques sont des habitudes et que la relation à l'invisible n'est pas consciente, ainsi beaucoup de gens ne savent pas pourquoi ils agissent ainsi. Ces pratiques sont donc situées aux frontières de l'habitude, de la simple envie de créer une ambiance et de l'interaction consciente avec un monde invisible.

De nombreuses idées circulent sur les djinns et influencent inévitablement la conception que les gens en ont et leur conduite. Mais ces courants d'idées sont multiples, variés et souvent contradictoires. Ceci pourrait partiellement expliquer pourquoi il n'y a pas une vérité unique, pourquoi chaque personne change son histoire selon le contexte, selon son expérience et sa capacité à 
I'exprimer. La plupart des Cairotes avec qui je parlais des djinns y croyaient et en doutaient, les redoutaient et s'en moquaient, étaient à la fois impliqués et sceptiques. Les éléments constitutifs des histoires sur les djinns créent des récits toujours partiels, personnels, contextuels et souvent incohérents.

\section{Crédibilité}

Pour le narrateur d'une histoire, il est très important d'intéresser et de retenir l'attention de ses auditeurs. C'est pourquoi les conteurs au Caire font un usage excessif des contrastes en privilégiant l'inattendu et les retournements de situations soudains. Les faits sont le plus souvent présentés comme exceptionnels, hors de la routine quotidienne. Plus ils insistent sur la réalité des faits («Wallâhi ${ }^{21}$, c'est vrai! »), plus ils suggèrent qu'ils sont incroyables et en accentuent le caractère insolite.

L'exagération est un trait caractéristique des histoires égyptiennes, elle fait partie de l'atmosphère propre à la narration. L'inclination pour l'exagération est même accentuée par les auditeurs qui, par leurs exclamations, leurs encouragements et leurs questions, poussent le narrateur aux limites du crédible. Beaucoup des histoires cairotes sont d'ailleurs proches de la blague et ont un effet humoristique. Une différence essentielle, toutefois, existe entre les blagues et les histoires : les blagues n'ont pas besoin d'être crédibles.

Une bonne histoire ne doit pas apparaître comme une invention, un mensonge ou une pure fantaisie, au risque de perdre l'attention de l'auditoire. Pour rendre une histoire crédible, les différents éléments constitutifs du récit doivent faire écho aux histoires, aux opinions et aux expériences passées des auditeurs. Les commentaires durant la narration questionnent souvent ce qui est crédible, ce qui ne l'est pas... « Non! C'est vrai? »Ces commentaires font partie de l'interaction, car le public met en question par ses exclamations la crédibilité de l'histoire et pousse le narrateur à se surpasser.

\section{Pour conclure : I'histoire comme une rencontre}

L'analyse des éléments constitutifs des histoires offre une source valable d'informations sur ce que les Cairotes pensent des djinns. Néanmoins, dans cette contribution, j'ai précisé que cette approche devait être complétée par une autre, interactionnelle. Ce n'est qu'en prenant en compte les commentaires des auditeurs, les différentes versions d'une histoire et leurs contextes, que l'on peut rendre compte des multiples messages qu'elle véhicule (les messages référentiels, intentionnels et moraux).

Avec cette approche, I'histoire et la narration sont comprises comme mode de connaissance plutôt que comme des moyens de communication. II faut

21. Pardieu! 
toujours nous interroger sur ce qui se passe durant la narration. Quelles sont les multiples intentions du narrateur? Quelles sont les différentes interprétations des auditeurs, sur le moment, quand ensuite ils commentent l'histoire et plus tard quand ils la racontent à d'autres?

Quand on analyse les histoires comme des ensembles d'événements (narration, écoute, réaction, transmission), leur intersubjectivité devient évidente. Par cette méthode, on voit que chaque histoire change selon le contexte dans lequel elle est racontée et que la valeur de chacune de ses versions ne repose pas seulement sur ses invariants. Une approche interactionnelle s'intéresse aux remaniements et aux différences dans la répétition. Cela signifie aussi que les répétitions ne correspondent pas nécessairement à des faits réels, mais que les mêmes éléments sont repris d'une version à une autre parce qu'ils ont un effet fort sur les auditeurs et assurent le succès de la narration.

Toucher son auditoire est la qualité d'une bonne histoire et d'un bon narrateur. Le savoir est transmis par les émotions, c'est un savoir qui n'est pas exprimé dans un langage discursif, mais qui affecte le cœur, les émotions et les comportements des auditeurs. Observer ce qui se passe pendant la narration d'une histoire permet de saisir les manipulations du narrateur, mais aussi les vertus de l'histoire qui, au-delà des intentions pratiques, a une valeur plus générale, morale.

Les histoires sur les djinns sont notamment formées de concepts et de croyances partagées qui assurent une crédibilité indispensable à leur succès. Quand de nouvelles idées sont intégrées à l'histoire, au cours de la narration, elles apportent des interprétations inattendues et alternatives sur les djinns. Ces idées pourront être réutilisées dans de futures histoires et, peut-être, influencer les conduites à venir.

\section{RÉFÉRENCES BIBLIOGRAPHIQUES}

Abdelsalem S., 1994, "L'Hôte divin : Étymologie du mot Zar », Nouvelle Revue d'Ethnopsychiatrie, 24, p. 73-90.

- 1995, «Le Zar musical et le Zar divinatoire : Description anthropologique des thérapeutiques traditionnelles au Soudan", Nouvelle Revue d'Ethnopsychiatrie, 28, p. 53-71.

ABu-LuGHOD L., 1997, "Islam and the Gendered Discourse of Death » dans Hopkins N. S. et Ibrahim S.E. (dirs), Arab Society: Class, Gender, Power and Development, Le Caire, The American University Press in Cairo, p. 527-550. Affergan F., 1997, La Pluralité des Mondes: Vers une autre anthropologie, Paris, Albin Michel.

Boddy J., 1989, Women and Alien Spirits : Women, Men and the Zár Cult in

Northern Sudan, Wisconsin, The University of Wisconsin Press.

BÖHRINGER-Thärigen G., 1996, Besessene Frauen : Der zâr-Kult von Omdurman, Wuppertal, Trickster im Peter Hammer Verlag. 
CéfAï D., 2003, L'Enquête de terrain, Paris, Éditions la Découverte.

Constantinides P., 1977, " III at Ease and Sick at Heart : Symbolic Behaviour in a Sudanese Healing Cult» dans Lewis I., (dir.), Symbols and Sentiments, Londres, Academic Press, p. 61-84.

Deleuze G. et Parnet C., 1991, Dialogen (traduit par Scheepers M.), Kampen, Kok Agora.

Devisch R., Stuer H. et Verrept H., 1989, " La signification psychoculturelle des plaintes gastriques », Santé Culture Health, 6 (1), p. 39-52.

Drieskens B., 2004, "The Misbehaviour of the Possessed : On Spirits, Morality and the Person » dans Dupret B. (dir.), Standing Trial: Law and the Person in the Modern Middle East, Londres, I.B. Tauris, p. 140-169.

- 2003, Living with Djinns : Understanding and Dealing with the Invisible in Cairo, Ph.D. Thesis, Université catholique de Louvain.

EARly E.A., 1982, "The logic of Well Being : Therapeutic Narratives in Cairo, Egypt », Social Science and Medicine, 16, p. 1491-1497.

- 1985, "Catharsis and Creation : The Everyday Narratives of Baladi Women of Cairo », Anthropological Quaterly, 58, p. 172-180.

- 1993, Baladi Women of Cairo, Playing with an Egg and a Stone, Le Caire, The American University in Cairo Press.

FAHD T., 1965, "Firâsa », Encyclopaedia of Islam II, nouvelle édition, Leyde, Brill, p. 916-917.

FrISHKOPF M., 1996, "La voix du poète : tarab et poésie dans le chant mystique soufi », Égypte/ Monde Arabe, n² 25, p. 85-117.

Gilsenan M., 1993, "Lying, Honor, and Contradiction » dans Bowen D.L. et EARLy E.A., Everyday Life in the Muslim Middle East, Bloomington et Indianopolis, Indiana University Press, p. 157-169.

Goffman E., 1973, La Mise en scène de la vie quotidienne :1 la présentation de soi, Paris, Les Éditions de Minuit.

Hanafi H., 1982, "The Relevance of the Islamic Alternative in Egypt », Arab Studies Quarterly, 4 (1/2), p. 54-74.

Hejalej M., 1996, Behind Closed Doors: Women's Oral Narratives in Tunis, New Brunswick, NJ, Rutgers University Press.

HIRSCHKIND C., 2001, "The ethics of listening : cassette-sermon auditions in contemporary Egypt », American Ethnologist, 28 (3), p. 623-649.

INHORn M.C., 1996, Infertility and Patriarchy : the Cultural Politics of Gender and Family Life in Egypt, Philadelphie, University of Pennsylvania Press.

KenYon S.M., 1995, "Zar as Modernization in Contemporary Sudan » dans KLASS M. et Weisgrau M. (dirs), Across the Boundaries of Belief : Contemporary Issues in the Anthropology of Religion, Boulder, Westview Press, p. 227247.

Mammoud S., 2001, «Feminist Theory, Embodiment, and the Docile Agent: Some Reflections on the Egyptian Islamic Revival », Cultural Anthropology, 16 (2), p. 202-236. 
Morsy S.A., 1978, "Sex Differences and Folk Illness in an Egyptian Village » dans Beck L. et Keddie N. (dirs), Women in the Muslim World, Cambridge et Londres, Harvard University Press, p. 599-616.

Nabhan M., 1994, Der zar-Kult in Ägypten: Rituelle Begegnung von Geist und Mensch: Ein Beispiel Komplementärer Glaubigkeit, Europäische Hochschulschriften, Frankfort/Main, Peter Lang.

Saunders L.W., 1977, "Variants in Zar Experience in an Egyptian Village » dans Crapazano V. et Garrison V. (dirs), Case Studies in Spirit Possession, New York/ Londres/Sydney/Toronto, John Wiley \& Sons, p. 177-191.

SChÖller M., 2001, "His Master's Voice: Gespräche mit Dschinnen im heutigen Ägypten », Die Welt des Islams, 41, 1, p. 32-71.

Sengers G., 2000, Vrouwen en Demonen: Zar en korangenezing in hedendaags Egypte, Amsterdam, Het Spinhuis.

Gobillot G., 2000, « La Conception originelle, ses interprétations et fonctions chez les penseurs musulmans », Annales Islamologiques, 18, Institut français d'Archéologie orientale.

Singerman D., 1995, Avenues of Participation : Family, Politics and Networks in Urban Quarters of Cairo, Princeton, Princeton University Press.

Starret G., 1998, Putting Islam to Work: Education, Politics and Religious Transformations in Egypt, Berkeley, University of California Press.

Watson H., 1992, Women in the City of the Dead, Londres, Hurst and Company.

WIKAN U., 1996, Tomorrow, God Willing: Self Made Destinies in Cairo, Chicago et Londres, The University of Chicago Press. 